\title{
Influence de la nature du concentré et du fourrage sur les interactions digestives chez le mouton
}

\author{
T Najar 1, H Oueslati ', N Moujehed 2, K Kraiem ' 2, D Sauvant ${ }^{3}$ \\ IINAT, 43 Av Charles Nicolle, Tunis, Tunisie ; ${ }^{2} E S A$ Mograne, Département de zootechnie, Mograne; \\ IINA Paris-Grignon, Département de zootechnie, 75005 Paris, France
}

\begin{abstract}
L'addition d'aliments concentrés aux fourrages distribués ad libitum à des ruminants modifie la digestibilité des constituants de la ration. Ces phénomènes constituent les interactions digestives entre aliments, elles ont été mises en évidence par plusieurs auteurs dont Dulphy et al (1983, Ann Zootech, 32, 53-80), Berge et Dulphy (1991, Ann Zootech, 40, 227-246).
\end{abstract}

Deux fourrages, à savoir un foin de vesce avoine $(F)$ et une paille de blé $(P)$ ont été associés dans les proportions de $0 \%, 30 \%$ et $60 \%$ à deux types d'aliments concentrés, "amidon" (CA) et "paroi" (CP) différents par leur teneur en paroi végétale, respectivement 7,3 et $13,4 \%$ d'ADF. Les 10 régimes ainsi constitués ont été distribués en deux repas par jour à 12 moutons en cages de digestibilité, répartis en 4 lots. Six mesures ont été effectuées par régime.

Des bilans fécaux ont été réalisés pendant 7 jours après une période d'adaptation de 15 jours. Les valeurs de la digestibilité de la matière organique ( $\mathrm{dMO}$ ) et de la fraction ligno-cellulosique (dADF) du régime ont été ajustées par le modèle :

$\mathrm{dMO}$ ou $\mathrm{dADF}=\mathrm{aC}^{2}+\mathrm{bC}+\mathrm{c}$

$C$ : proportion de MO ou de ADF apportée par le concentré

$a, b, c:$ constantes du modèle

L'ingestion moyenne de matière sèche a été de $1599 \mathrm{~g} / \mathrm{j}$ et de $1181 \mathrm{~g} / \mathrm{j}$ respectivement pour les rations à base de foin et de paille. Les ajustements significatifs de la dMO à la proportion de concentré sont rapportés cidessous. Les dMO du foin et de la paille sont de 0,55 et 0,51 respectivement. La signification statistique du coefficient (a) du modèle révèle l'existence d'une interaction digestive négative dans le cas des régimes $\mathrm{F}+\mathrm{CA}, \mathrm{P}+\mathrm{CA}$ et $P+C P$. Cependant, pour le régime $F+C P$, la variation de la digestibilité du fourrage avec le niveau de concentré est linéaire. L'extrapolation des équations d'ajustement à $\mathrm{C}=1$ permet d'estimer la digestibilité des aliments concentrés à $86 \%$ pour $\mathrm{CA}$ et $67 \%$ pour $\mathrm{CP}$.

La variation du CUD du fourrage associée à $0 \%$ ou $60 \%$ de concentré montre que la dMO n'est pas modifiée significativement lorsque le foin est associé au CP ; en revanche elle diminue de 17,5 points avec le CA. Elle diminue de 7,8 points et 20 points respectivement pour la paille lorsqu'elle est associée au CA ou au CP. L'ajustement statistique des dADF montre que les coefficients du terme quadratique ne sont significatifs que pour les régimes $\mathrm{F}+\mathrm{CA}$ et $\mathrm{P}+\mathrm{CP}$.

Ces résultats confirment ceux rapportés par Berge et Dulphy (1991), et Archimède (1992, Thèse doctorat INA P-G, France). II est à noter que pour les fourrages pauvres, ces auteurs rapportent une interaction digestive positive avec l'aliment concentré lorsque le niveau de celui-ci est faible $(<30 \%)$, celle-ci serait liée à l'effet de l'apport azoté du concentré. L'interaction négative observée dans ce travail, malgré une faible teneur en MAT des régimes à base de paille, serait liée à l'importance de la proportion de concentré dans la ration ainsi qu'à une meilleure digestibilité des fourrages.

\begin{tabular}{|c|c|c|c|c|c|c|c|}
\hline Fourrage & concentré & sign. modèle 1 & a & $b$ & c & $\mathrm{R}$ & ETR \\
\hline $\mathrm{F}$ & $\mathrm{CA}$ & $\star \star$ & $29.10^{-6}$ & - & 0,55 & 0,61 & 5,7 \\
\hline $\mathrm{F}$ & $\mathrm{CP}$ & $\star \star$ & - & 0,0011 & 0,55 & 0,55 & 4,5 \\
\hline $\mathrm{P}$ & $\mathrm{CA}$ & $\star \star \star$ & $33.10^{-6}$ & - & 0,51 & 0,76 & 4,8 \\
\hline P & $\mathrm{CP}$ & ** & $19.10^{-6}$ & - & 0,50 & 0,57 & 4,6 \\
\hline
\end{tabular}

